\title{
GENERALIZED WEYL CONFORMAL CURVATURE TENSOR OF GENERALIZED RIEMANNIAN SPACE
}

\author{
NENAD O. VESIĆ \\ Received 14 November, 2017
}

\begin{abstract}
In this paper certain invariants of conformal mappings of the generalized Riemannian space are obtained. Here are the general results. These invariants are the analogues of the Thomas projective parameter and generalized Weyl projective tensor.
\end{abstract}

2010 Mathematics Subject Classification: 14L24; 14A22; 53A30; 53B20

Keywords: equation, Weyl conformal curvature tensor, conformal mapping, generalized Riemannian space, invariant

\section{INTRODUCTION AND MOTIVATION}

A differentiable $N$-dimensional manifold on which the non-symmetric basic tensor $G_{i j}$ is defined is a generalized Riemannian space. Because of the non-symmetry $G_{i j} \neq G_{j i}$, it is possible to determine the symmetric and antisymmetric part of the metric tensor $G_{i j}$ :

$$
g_{i j}=\frac{1}{2}\left(G_{i j}+G_{j i}\right) \quad \text { and } \quad F_{i j}=\frac{1}{2}\left(G_{i j}-G_{j i}\right) .
$$

Metric $g$ is non-degenerate, i.e. the matrix $\left(g_{i j}\right)_{N \times N}$ is non-singular. The coordinates of contravariant metric tensor $g$ of associated space $\mathbb{R}_{N}$ are the corresponding inverse matrix elements $\left(g^{i j}\right)=\left(g_{i j}\right)^{-1}$. It also holds that: $g^{i \alpha} g_{j \alpha}=\delta_{j}^{i}$.

The use of a non-symmetric basic tensor and a non-symmetric affine connection is motivated by Einstein's and Eisenhart's works [5-7]. In Einstein's Unified Field Theory, the symmetric part $g_{i j}$ of the basic tensor $G_{i j}$ is related to gravitation, and the anti-symmetric part $F_{i j}$ is related to electromagnetism. Some mathematicians have studied and developed the theory of non-symmetric affine connection spaces and the theory of generalized Riemannian spaces, for example, L. P. Eisenhart [7], S. M. Minčić [14, 15], M. Prvanović [17]. 
Affine connection coefficients of the space $\mathbb{G} \mathbb{R}_{N}$ are (generalized) Christoffel symbols of the second kind

$$
\Gamma_{j k}^{i}=\frac{1}{2} g^{i \alpha}\left(G_{j \alpha, k}-G_{j k, \alpha}+G_{\alpha k, j}\right),
$$

where the partial derivative $\partial / \partial x^{i}$ is marked with a comma. In general, $\Gamma_{j k}^{i} \neq \Gamma_{k j}^{i}$ is valid. This coefficient is decomposed in two parts, the symmetric part $\stackrel{0}{\Gamma}_{j k}^{i}$ and the anti-symmetric part $T_{j k}^{i}$, where

$$
\stackrel{0}{\Gamma}_{j k}^{i}=\frac{1}{2}\left(\Gamma_{j k}^{i}+\Gamma_{k j}^{i}\right) \text { and } T_{j k}^{i}=\frac{1}{2}\left(\Gamma_{j k}^{i}-\Gamma_{k j}^{i}\right) .
$$

The symmetric part $\stackrel{0}{\Gamma}_{j k}^{i}$ is the Levi-Civita affine connection of the symmetric metric $g$ and the anti-symmetric part $T_{j k}^{i}$ is called the torsion tensor.

On the basis of a non-symmetric affine connection, it is possible to consider four types of covariant derivatives with regard to the affine connection of the space $\mathbb{G} \mathbb{R}_{N}$ (see [14, 15]). Afterwards, a family of $\mathbb{G} \mathbb{R}_{N}$ curvature tensors was obtained

$$
R_{j m n}^{i}=\stackrel{0}{R}_{j m n}^{i}+u T_{j m ; n}^{i}+u^{\prime} T_{j n ; m}^{i}+v T_{j m}^{\alpha} T_{\alpha n}^{i}+v^{\prime} T_{j n}^{\alpha} T_{\alpha m}^{i}+w T_{m n}^{\alpha} T_{\alpha j}^{i},
$$

where $u, u^{\prime}, v, v^{\prime}, w$ are real constants, a covariant derivative based on an affine connection $\stackrel{0}{\Gamma}$ is denoted by a semicolon and a curvature tensor

$$
\stackrel{0}{R}_{j m n}^{i}=\stackrel{0}{\Gamma}_{j m, n}^{i}-\stackrel{0}{\Gamma}_{j n, m}^{i}+\stackrel{0}{\Gamma}_{j m}^{\alpha} \stackrel{0}{\Gamma}_{\alpha n}^{i}-\stackrel{0}{\Gamma}_{j n}^{\alpha} \stackrel{0}{\Gamma}_{\alpha m}^{i} .
$$

Geometrical objects that are invariant with respect to conformal mappings play an important role in the theory of gravity $[9,22]$. The aim of our paper is to generalize invariants of conformal mappings from [2,19]. These mappings generalize the concept of conformal mappings defined by N. S. Sinyukov [18], J. Mikeš [11-13], S. E. Stepanov [20,21].

\section{CONFORMAL MAPPINGS OF GENERALIZED RIEMANNIAN SPACE}

Suppose

$$
f: \mathbb{G} \mathbb{R}_{N} \rightarrow \mathbb{G} \overline{\mathbb{R}}_{N}
$$

is a diffeomorphism. We can consider the manifolds $\mathbb{G} \mathbb{R}_{N}$ and $\mathbb{G} \mathbb{R}_{N}$ in the common system of local coordinates with respect to this mapping (see Figure 1). Namely, if $f$ maps a point $M \in \mathbb{G}_{N}$ to point $\bar{M} \in \mathbb{G}_{N}$ and if $(U, \varphi)$ is a local chart around the point $M$ it will be $\varphi(M)=x=\left(x^{1}, \cdots, x^{N}\right) \in \mathbb{E}^{N}$ (Euclidean $N$-space). In this case, we define (for the coordinate mapping in the $\mathbb{G}_{\mathbb{R}}$ ) the mapping $\bar{\varphi}=\varphi \circ f^{-1}$, and then

$$
\bar{\varphi}(\bar{M})=\left(\varphi \circ f^{-1}\right)(f(M))=\varphi(M)=x=\left(x^{1}, \cdots, x^{N}\right) \in \mathbb{E}^{N} .
$$


Therefore the points $M$ and $\bar{M}=f(M)$ have the same local coordinates.

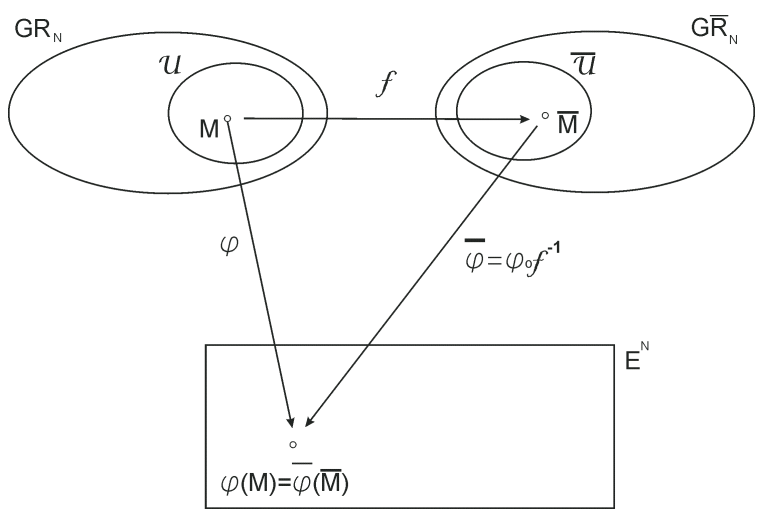

FIGURE 1. Common system of coordinates

Based on Eisenhart's results [7], many authors started the researches on conformal mappings between Riemannian and generalized Riemannian spaces as well as invariants of these mappings (see [1-4, 8, 10-13,16-21,23]).

One says that diffeomorphism $f: \mathbb{G}_{N} \rightarrow \mathbb{G}_{N}$ is conformal mapping [19] if the metric tensors $G_{i j}$ and $\bar{G}_{i j}$ of the spaces $\mathbb{G}_{N}$ and $\mathbb{G} \overline{\mathbb{R}}_{N}$ satisfy the condition

$$
\bar{G}_{i j}=e^{2 \psi} G_{i j},
$$

where $\psi$ is a scalar function. The basic equation of conformal mapping $f$ is

$$
\bar{\Gamma}_{j k}^{i}=\Gamma_{j k}^{i}+\psi_{j} \delta_{k}^{i}+\psi_{k} \delta_{j}^{i}-\psi^{i} g_{j k}+\xi_{j k}^{i},
$$

for $\psi_{i}=\partial \psi / \partial x^{i}, \psi^{i}=g^{i \alpha} \psi_{\alpha}$, and tensor $\xi_{j k}^{i}$ anti-symmetric in the indices $j$ and $k$. After symmetrizing the equation (2.3) by indices $j$ and $k$ and contracting the symmetrized equation by indices $i$ and $k$, we obtain that is

$$
\psi_{j}=\frac{1}{N}\left(\frac{0}{\Gamma}{ }_{j \alpha}^{\alpha}-\stackrel{0}{\Gamma}_{j \alpha}^{\alpha}\right)
$$

This result proves that it holds

$$
\begin{aligned}
\frac{0}{\Gamma}_{j k}^{i}-\stackrel{0}{\Gamma}_{j k}^{i}= & \frac{1}{N}\left(\bar{\Gamma}_{j \alpha}^{\alpha} \delta_{k}^{i}+\stackrel{0}{\Gamma}_{k \alpha}^{\alpha} \delta_{j}^{i}-\stackrel{0}{\Gamma}_{\alpha \beta}^{\beta} g^{i \alpha} g_{j k}\right) \\
& -\frac{1}{N}\left(\stackrel{0}{\Gamma}_{j \alpha}^{\alpha} \delta_{k}^{i}+\stackrel{0}{\Gamma}_{k \alpha}^{\alpha} \delta_{j}^{i}-\stackrel{0}{\Gamma}_{\alpha \beta}^{\beta} g^{i \alpha} g_{j k}\right)
\end{aligned}
$$


Weyl conformal curvature tensor

$$
\begin{aligned}
\stackrel{0}{C}_{j m n}^{i}= & \stackrel{0}{R_{j m n}^{i}}+\frac{1}{N-2}\left(\delta_{n}^{i} \stackrel{0}{R_{j m}}-\delta_{m}^{i} \stackrel{0}{R_{j n}}+\stackrel{0}{R_{n}^{i}} g_{j m}-\stackrel{0}{R}_{m}^{i} g_{j n}\right) \\
& +\frac{\stackrel{0}{R}}{(N-1)(N-2)}\left(\delta_{m}^{i} g_{j n}-\delta_{n}^{i} g_{j m}\right),
\end{aligned}
$$

is an invariant of the mapping $f$.

After anti-symmetrizing the equation (2.3) by indices $j$ and $k$, we obtain that is $\xi_{j k}^{i}=\bar{T}_{j k}^{i}-T_{j k}^{i}$. If one substitutes this equality in the equation (2.3), the nonsymmetric affine connection of the space $\mathbb{G R}_{N}$ will be reduced to the symmetric affine connection $\stackrel{0}{\Gamma}$. For this reason, different authors have studied equitorsion conformal mappings (the case of $\xi_{j k}^{i}=0$ ) or conformal mappings of special generalized Riemannian spaces $[16,19,23]$. We will generalize the existing invariants of conformal mappings in this paper.

\section{GENERALIZED INVARIANTS OF CONFORMAL MAPPINGS}

From the equation (2.2), we get

$$
\bar{g}_{i j}=e^{2 \psi} g_{i j}, \quad \bar{F}_{i j}=e^{2 \psi} F_{i j}, \quad \bar{g}^{i j}=e^{-2 \psi} g^{i j},
$$

which proves that is

$$
\bar{g}^{i j} \bar{g}_{m n}=g^{i j} g_{m n} \quad \text { and } \quad \bar{g}^{i j} \bar{F}_{m n}=g^{i j} F_{m n} .
$$

With regard to the equation (2.5), we obtain that it holds

$$
\frac{0}{\Gamma}_{j k}^{i}-\stackrel{0}{\Gamma}_{j k}^{i}=\bar{\zeta}_{j k}^{i}-\zeta_{p}^{i}
$$

$p=1,2$, for

$$
\zeta_{1}^{i}=\stackrel{0}{\Gamma}_{j k}^{i} \quad \text { and } \quad \zeta_{2}^{i} j k=\frac{1}{N}\left(\stackrel{0}{\Gamma}_{j \alpha}^{\alpha} \delta_{k}^{i}+\stackrel{0}{\Gamma}_{k \alpha}^{\alpha} \delta_{j}^{i}-\stackrel{0}{\Gamma}_{\alpha \beta}^{\beta} g^{i \alpha} g_{j k}\right),
$$

and the corresponding $\bar{\zeta}_{p}^{i}{ }_{j k}$.

From the equations $(1.2,1.3)$ and the equality $g_{; k}^{i j}=0$, one obtains that the torsion tensor $T_{j k}^{i}$ may be expressed as

$$
\begin{aligned}
T_{j k}^{i} & =\frac{1}{2} g^{i \alpha}\left(F_{j \alpha, k}-F_{j k, \alpha}+F_{\alpha k, j}\right) \\
& =\frac{1}{2}\left(\left(g^{i \alpha} F_{j \alpha}\right)_{; k}-\left(g^{i \alpha} F_{k \alpha}\right)_{; j}-\left(g^{i \alpha} F_{j k}\right)_{; \alpha}\right) .
\end{aligned}
$$


Based on this equation and the second of the equalities (3.1), one establishes that it holds

$$
\bar{T}_{j k}^{i}-T_{j k}^{i}=\bar{\tau}_{r k}^{i}-\tau_{r}^{i}{ }_{j k}
$$

$r=\left(r_{1}, r_{2}, r_{3}, r_{4}, r_{5}\right), r_{1}, \ldots, r_{5} \in\{1,2\}$, for

$$
\tau_{r}^{i}{ }_{j k}^{i}=\frac{1}{2} g^{\alpha \beta}\left(\zeta_{r_{1}}^{i}{ }_{\beta k} F_{j \alpha}-\zeta_{r_{2}}^{i}{ }_{\beta j} F_{k \alpha}-\zeta_{r_{3}}^{i} \alpha \beta F_{j k}+\delta_{\beta}^{i} \zeta_{r_{4}}^{\gamma}{ }_{j \alpha} F_{\gamma k}-\delta_{\beta}^{i} \zeta_{r_{5}}^{\gamma}{ }_{k \alpha} F_{j \gamma}\right)
$$

and the corresponding $\bar{\tau}_{r k}^{i}$.

Plugging the equations $(2.4,3.5)$ into the basic equation $(2.3)$, we get

$$
\underset{p^{j k}}{\overline{\mathcal{T}}^{i}}=\underset{p^{j k}}{\mathcal{T}^{i}},
$$

for

$$
\underset{r}{\mathcal{T}_{p}^{i}}{ }^{j k}=\Gamma_{j k}^{i}-\zeta_{p}^{i}{ }_{j k}-\tau_{r}^{i}{ }^{j}
$$

the above defined $\zeta_{p}^{i}{ }_{j k}^{i}, \tau_{r j k}^{i}$ and the corresponding $\underset{p^{j}}{\overline{\mathcal{T}}^{i}}$.

It holds the following lemma:

Lemma 1. Let $f: \mathbb{G} \mathbb{R}_{N} \rightarrow \mathbb{G} \overline{\mathbb{R}}_{N}$ be a conformal mapping. The set of geometrical object given by the equation (3.6) is the set of invariants of the mapping $f$.

Corollary 1. Let $f: \mathbb{G} \mathbb{R}_{N} \rightarrow \mathbb{G}_{N}$ be an equitorsion conformal mapping. The geometrical object

$$
\stackrel{0}{\mathcal{T}}_{j k}^{i}=\stackrel{0}{\Gamma}_{j k}^{i}-\zeta_{2}^{i}{ }_{j k}
$$

is an invariant of the mapping $f$.

The families $R_{j m n}^{i}$ and $\bar{R}_{j m n}^{i}$ of curvature tensors of the spaces $\mathbb{G} \mathbb{R}_{N}$ and $\mathbb{G} \overline{\mathbb{R}}_{N}$ satisfy the equation

$$
\begin{aligned}
\bar{R}_{j m n}^{i}-R_{j m n}^{i}= & \bar{R}_{j m n}^{i}-\stackrel{0}{R}_{j m n}^{i}+u\left(\bar{T}_{j m \overline{ }}^{i}-T_{j m ; n}^{i}\right) \\
& +u^{\prime}\left(\bar{T}_{j n \overline{ }}^{i}-T_{j n ; m}^{i}\right)+v\left(\bar{T}_{j m}^{\alpha} \bar{T}_{\alpha n}^{i}-T_{j m}^{\alpha} T_{\alpha n}^{i}\right) \\
& +v^{\prime}\left(\bar{T}_{j n}^{\alpha} \bar{T}_{\alpha m}^{i}-T_{j n}^{\alpha} T_{\alpha m}^{i}\right)+w\left(\bar{T}_{m n}^{\alpha} \bar{T}_{\alpha j}^{i}-T_{m n}^{\alpha} T_{\alpha j}^{i}\right) .
\end{aligned}
$$


With regard to the invariance $\overline{0}_{j m n}^{i}=\stackrel{0}{C}_{j m n}^{i}$, we obtain that is

$$
\begin{aligned}
\frac{0}{R_{j m n}^{i}}-\stackrel{0}{R}_{j m n}^{i}= & \frac{1}{N-2}\left(\delta_{n}^{i} \stackrel{0}{R}_{j m}-\delta_{m}^{i} \stackrel{0}{R}_{j n}+\stackrel{0}{R}_{n}^{i} g_{j m}-\stackrel{0}{R}_{m}^{i} g_{j n}\right) \\
& -\frac{1}{N-2}\left(\delta_{n}^{i} \frac{0}{R_{j m}}-\delta_{m}^{i} \stackrel{0}{R}_{j n}+\stackrel{0}{R}_{n}^{i} \bar{g}_{j m}-\stackrel{0}{R}_{m}^{i} \bar{g}_{j n}\right) \\
& +\frac{1}{(N-1)(N-2)}\left(\stackrel{0}{R}\left(\delta_{m}^{i} g_{j n}-\delta_{n}^{i} g_{j m}\right)-{ }_{\bar{R}}\left(\delta_{m}^{i} \bar{g}_{j n}-\delta_{n}^{i} \bar{g}_{j m}\right)\right)
\end{aligned}
$$

From the equations $(3.2,3.5)$, we establish that

$$
\begin{aligned}
& \bar{T}_{j m ; n}^{i}-T_{j m ; n}^{i}={\underset{s}{s}{ }_{r}^{j}}_{j m n}^{i}-\underset{r}{\sigma_{j}^{i}} i m n
\end{aligned}
$$

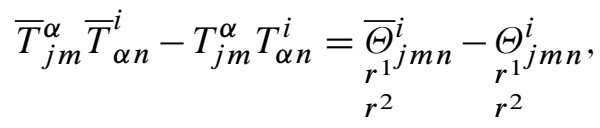

for $s=\left(s_{1}, s_{2}, s_{3}\right), r=\left(r_{1}, \ldots, r_{5}\right), r^{u}=\left(r_{1}^{u}, \ldots, r_{5}^{u}\right), r_{v}, r_{v}^{u}, s_{1}, s_{2}, s_{3} \in\{1,2\}$,

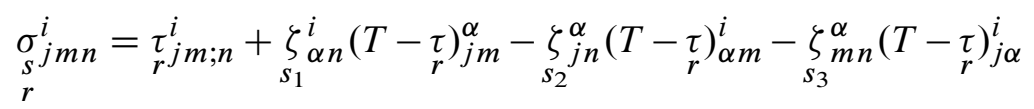

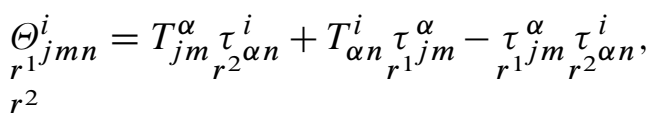

and the corresponding $\underset{r}{\sigma_{j}^{j}} \bar{\sigma}_{j m n}^{i}$ and $\underset{r^{2}}{\bar{\Theta}^{2}}{ }_{j m n}^{i}$.

From equation (1.5) it turns out that:

$$
\begin{aligned}
& \stackrel{0}{R}_{j m n}^{i}=R_{j m n}^{i}-u T_{j m ; n}^{i}-u^{\prime} T_{j n ; m}^{i}-v T_{j m}^{\alpha} T_{\alpha n}^{i}-v^{\prime} T_{j n}^{\alpha} T_{\alpha m}^{i}-w T_{m n}^{\alpha} T_{\alpha j}^{i}, \\
& \stackrel{0}{R}_{i j}=R_{i j}-u T_{i j ; \alpha}^{\alpha}-\left(v^{\prime}+w\right) T_{i \beta}^{\alpha} T_{\alpha j}^{\beta}, \\
& \stackrel{0}{R_{j}^{i}}=R_{j}^{i}-u g^{i \alpha} T_{\alpha j ; \beta}^{\beta}-\left(v^{\prime}+w\right) g^{i \alpha} T_{\alpha \gamma}^{\beta} T_{\beta j}^{\gamma}, \\
& \stackrel{0}{R}=R-\left(v^{\prime}+w\right) T_{\gamma \beta}^{\alpha} T_{\alpha \delta}^{\beta} g^{\gamma \delta},
\end{aligned}
$$

for Ricci-tensor $R_{i j}=R_{i j \alpha}^{\alpha}$, mixed Ricci-curvature tensor $R_{j}^{i}=g^{i \alpha} R_{j \alpha}$ and the scalar curvature $R=R_{\alpha}^{\alpha}$.

After introducing the equations (3.9-3.17) into the equation (3.8), one obtains that

$$
\bar{C}_{\rho}^{i}{ }_{j m n}=\underset{\rho}{C}{ }_{j m n}^{i},
$$


for

$$
\begin{aligned}
& C_{\rho}^{i}{ }_{j m n}=R_{j m n}^{i}+\frac{1}{N-2}\left(\delta_{m}^{i} R_{j n}-\delta_{n}^{i} R_{j m}+R_{m}^{i} g_{j n}-R_{n}^{i} g_{j m}\right) \\
& +\frac{R}{(N-1)(N-2)}\left(\delta_{m}^{i} g_{j n}-\delta_{n}^{i} g_{j m}\right) \\
& -\frac{u}{N-2}\left(\delta_{m}^{i} T_{j n ; \alpha}^{\alpha}-\delta_{n}^{i} T_{j m ; \alpha}^{\alpha}+g^{i \alpha} T_{\alpha m ; \beta}^{\beta} g_{j n}-g^{i \alpha} T_{\alpha n ; \beta}^{\beta} g_{j m}\right) \\
& -\frac{v^{\prime}+w}{N-2}\left(\delta_{m}^{i} T_{j \beta}^{\alpha} T_{\alpha n}^{\beta}-\delta_{n}^{i} T_{j \beta}^{\alpha} T_{\alpha m}^{\beta}+g^{i \alpha} T_{\alpha \gamma}^{\beta}\left(T_{\beta m}^{\gamma} g_{j n}-T_{\beta n}^{\gamma} g_{j m}\right)\right) \\
& -\frac{v^{\prime}+w}{(N-1)(N-2)} T_{\gamma \beta}^{\alpha} T_{\alpha \delta}^{\beta} g^{\gamma \delta}\left(\delta_{m}^{i} g_{j n}-\delta_{n}^{i} g_{j m}\right)
\end{aligned}
$$

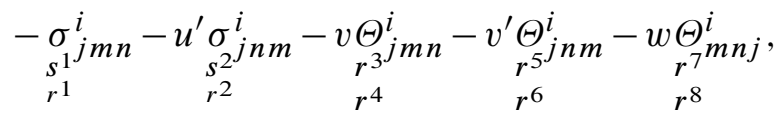

$\rho=\left(s^{1}, s^{2}, r^{1}, \ldots, r^{8}\right)$, and the corresponding $\bar{C}_{\rho}^{i} j m n$.

The following theorem holds:

Theorem 1. Let $f: \mathbb{G} \mathbb{R}_{N} \rightarrow \mathbb{G} \overline{\mathbb{R}}_{N}$ be a conformal mapping of generalized Riemannian space $\mathbb{G} \mathbb{R}_{N}$. The families $C_{\rho}^{i}{ }_{j m n}$ of geometrical objects given by the equation (3.18) are families of invariants of the conformal mapping $f$.

Corollary 2. Let $f: \mathbb{G} \mathbb{R}_{N} \rightarrow \mathbb{G} \overline{\mathbb{R}}_{N}$ be an equitorsion conformal mapping. The family of geometrical objects

$$
\begin{aligned}
& \widetilde{\widetilde{r}}_{j}^{i}{ }_{j m n}=R_{j m n}^{i}+\frac{1}{N-2}\left(\delta_{m}^{i} R_{j n}-\delta_{n}^{i} R_{j m}+R_{m}^{i} g_{j n}-R_{n}^{i} g_{j m}\right)
\end{aligned}
$$

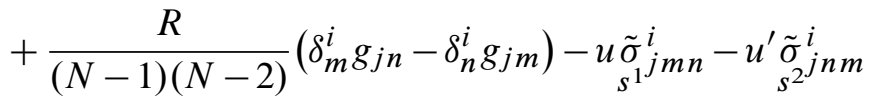

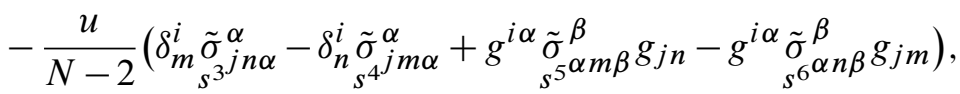

for

$$
\tilde{\sigma}_{s^{k}}^{i} j m n=\zeta_{s_{1}^{k}}^{i} T_{j m}^{\alpha}-\zeta_{s_{2}^{k}}^{\alpha} T_{\alpha m}^{i}-\zeta_{s_{3}^{k}}^{\alpha}{ }_{m n}^{\alpha} T_{j \alpha}^{i},
$$

$k=1, \ldots, 6$, is the family of invariants of the mapping $f$.

\section{CONCLUSION}

Weyl conformal curvature tensor was generalized in this paper. We also obtained invariants of conformal mappings analogous to the Thomas projective parameter. 


\section{ACKNOWLEDGEMENTS}

This paper is financially supported by Serbian Ministry of Education, Science and Technological Development, Grant No. 174012.

The author express his gratitude to the anonymous referees for their time dedicated to the review of this manuscript.

\section{REFERENCES}

[1] H. M. Abood, “Almost Hermitian Manifold with Flat Bochner Tensor.” Eur. J. Pure Appl. Math., vol. 3, no. 4, pp. 730-736, 2010.

[2] S. Bochner, "Curvature and Betti Numbers. II." Ann. Math. (2), vol. 50, no. 1, pp. 77-93, 1949, doi: $10.2307 / 1969353$.

[3] H. Chuda and J. Mikeš, "Conformally geodesic mappings satisfying a certain initial condition," Arch. Math., Brno, vol. 47, no. 5, pp. 389-394, 2011.

[4] H. Chuda and M. Shiha, "Conformal holomorphically projective mappings satisfying a certain initial condition," Miskolc Math. Notes, vol. 14, no. 2, pp. 569-574, 2013.

[5] A. Einstein, "A generalization of the relativistic theory of gravitation." Ann. Math. (2), vol. 46, no. 4, pp. 578-584, 1945, doi: 10.2307/1969197.

[6] A. Einstein, "Bianchi Identities in the Generalized Theory of Gravitation." Can. J. Math, vol. 2, pp. 120-128, 1950.

[7] L. P. Eisenhart, Riemannian Geometry. New York: Princeton University Press, 1967.

[8] L. E. Evtushik, I. Hinterleitner, N. I. Guseva, and J. Mikeš, "Conformal mappings onto Einstein spaces," Russ Math., vol. 60, no. 10, pp. 5-9, 2016, doi: 10.3103/S1066369X16100029.

[9] E. Goulart and M. Novello, "Cosmological Stability of Weyl Conformal Tensor," Gravit. Cosmol., vol. 14, no. 4, pp. 321-326, 2008.

[10] S. B. Mathur, "Conformal spaces and curvature tensors," Indian J. Pure Appl. Math., vol. 2, pp. 29-31, 1971

[11] J. Mikeš, V. Kiosak, and A. Vanžurová, Geodesic mappings of manifolds with affine connection. Olomouc: Palacky University, 2008.

[12] J. Mikeš, E. Stepanova, A. Vanžurová, and et al., Differential Geometry of Special Mappings. Olomouc: Palacky University, 2015.

[13] J. Mikeš, A. Vanžurová, and I. Hinterleitner, Geodesic Mappings and Some Generalizations. Olomouc: Palacky University, 2009.

[14] S. M. Minčić, "Ricci identities in the space of non-symmetric affine connection." Mat. Vesnik, vol. 10 (25), no. 2, pp. 161-172, 1973.

[15] S. M. Minčić, "Independent curvature tensors and pseudotensors of spaces with non-symmetric affine connexion.” Colloq. Math. Soc. Janos Bolyai, vol. 31, pp. 445-460, 1979.

[16] M. S. Najdanović, M. L. Zlatanović, and I. Hinterleitner, "Conformal and Geodesic Mappings of Generalized Equidistant Spaces.” Publ. Inst. Math., Nouv. Ser, vol. 98, no. 112, pp. 71-84, 2015, doi: 10.2298/PIM1512071N.

[17] M. Prvanović, "Projective and conformal transformations in recurrent and Ricci-recurrent Riemannian space.” Tensor, New Ser., vol. 12, no. 3, pp. 219-226, 1962.

[18] N. S. Sinyukov, Geodesic Mappings of Riemannian Spaces, (in Russian). Moskva: Nauka, 1979.

[19] M. S. Stanković, L. S. Velimirović, S. M. Minčić, and M. L. Zlatanović, "Equitorsion Conform Mappings of Generalized Riemannian Spaces.” Mat. Vesnik, vol. 61, no. 2, pp. 119-129, 2009.

[20] S. E. Stepanov, I. A. Alexandrova, I. I. Tsyganok, and J. Mikeš, "Conformal Killing forms on totally umbilical submanifolds." J. Math. Sci., New York, vol. 217, no. 5, pp. 525-539, 2016, doi: 10.1007/s10958-016-2989-5. 
[21] S. E. Stepanov and I. I. Tsyganok, "Theorems of existence and non-existence of conformal Killing forms." zv. Vyssh. Uchebn. Zaved. Mat., no. 10, pp. 54-61, 2014.

[22] H. Zhang, Y. Zhang, and X.-Z. Li, "Dynamical spacetimes in conformal gravity." Nucl. Phys., B, vol. 921, pp. 522-537, 2017.

[23] M. Zlatanović, I. Hinterleitner, and M. Najdanović, "On equitorsion concircular tensors of generalized Riemannian spaces.” Filomat, vol. 28, no. 3, pp. 463-471, 2014, doi: 10.2298/FIL1403463Z.

\section{Author's address}

Nenad O. Vesić

University of Nish, Faculty of Science and Mathematics, Višegradska 33, 18000 Niš, Serbia

E-mail address: n.०.vesic@out look.com 